\title{
Altered expression of Krüppel-like factor 4 and $\beta$-catenin in human gastric cancer
}

\author{
N. ZHANG ${ }^{*}$, J.ZHANG ${ }^{*}$, Z.W.WANG, L. ZHA and Z. HUANG \\ Department of General Surgery, The First Affiliated Hospital of Chongqing Medical University, \\ Chongqing 400016, P.R. China
}

Received December 10, 2011; Accepted February 10, 2012

DOI: $10.3892 / \mathrm{ol} .2012 .619$

\begin{abstract}
The effects of the interaction between KLF4 and $\beta$-catenin may be significant in human carcinogenesis and tumor development. This study aimed to determine whether the expression of KLF4 and $\beta$-catenin in gastric cancer tissues is associated with clinicopathological characteristics. Western blot analysis and immunohistochemistry were performed to detect KLF4 and $\beta$-catenin expression in tumor and corresponding non-cancerous tissues from 49 patients. The data revealed that KLF4 expression was significantly reduced in gastric cancer tissues compared with normal tissues. By contrast, the expression of the $\beta$-catenin protein was significantly increased in all tumor tissues, but was not expressed in distant normal mucosae. The altered expression of the KLF4 and $\beta$-catenin proteins was associated with advanced tumor stage and gastric cancer. In addition, the expression of the KLF4 and $\beta$-catenin proteins was inversely associated in moderately differentiated human gastric cancers. This study showed that $\beta$-catenin expression is significantly increased and KLF4 protein expression is markedly decreased in gastric cancer tissues, thus showing that the expression of KLF4 is inversely correlated with that of $\beta$-catenin in gastric cancer. The altered expression of the two proteins is associated with advanced tumor stage in gastric cancer.
\end{abstract}

\section{Introduction}

Gastric cancer remains the fourth most common malignancy and the second leading cause of cancer-related mortality worldwide, despite a steady decline in incidence over the past several decades (1). In recent years, the survival rate of

Correspondence to: Dr Zi-Wei Wang, Department of General Surgery, The First Affiliated Hospital, Chongqing Medical University, No. 1 Youyi Rd., Yuzhong District, Chongqing 400016, P.R. China

E-mail: wangziwei571@hotmail.com

*Contributed equally

Key words: Krüppel-like factor 4, $\beta$-catenin, immunohistochemistry, tumor cell differentiation gastric cancer has significantly improved due to advances in treatments, including surgery, chemotherapy and radiotherapy. However, approximately 800,000 individuals still succumb to gastric carcinoma each year worldwide. Clinically, early gastric cancer is often asymptomatic or causes non-specific symptoms; when symptoms occur, the cancer has often reached an advanced stage. This results in the poor short-term survival rate of gastric cancer patients due to primary tumor invasion and metastasis (2). Similar to other types of cancer, tumor invasion and metastasis are serious clinical problems and are the most notable properties of aggressive gastric carcinoma. Gastric cancer development is often associated with a number of molecular abnormalities, including the inactivation of various tumor suppressor genes and/or activation of various oncogenes $(3,4)$. A number of these genes have been investigated as biological markers for the prediction of gastric cancer staging and lymph node metastasis, but the potential roles of these genes in the etiology of gastric cancer remain poorly understood. Investigations into the molecular alterations in gastric cancer may provide novel insights into the mechanisms responsible for stomach carcinogenesis and lead to the development of biomarkers for the early detection of gastric cancer and the prediction of its prognosis.

Krüppel-like factor 4, (KLF4) is a newly identified zinc-finger transcription factor (5). Similar to all Krüppel-like factors, KLF4 has three zinc-finger domains in its C-terminus and is involved in various cell and developmental processes, including cell terminal differentiation and carcinogenesis (6). The results of previous studies have shown that the expression level of KLF4 is high in the epithelial cells of the skin, lung and gastrointestinal tract and is particularly elevated in terminally differentiated and postmitotic epithelial cells (7). Other studies have found that KLF4 expression is associated with the growth arrest of cultured cells (8) and KLF4 overexpression reduces colorectal cancer colony formation, migration and invasion. By contrast, a decrease or loss of KLF4 expression frequently occurs in various types of cancer, including cancers of the colorectum $(9,10)$, stomach $(11)$, esophagus (12), prostate (13) and lung (14). The expression levels of KLF4 were found to be inversely associated with the size of intestinal adenoma in animal experiments (11). In addition, the results of studies have shown that a decreased KLF4 expression is associated with poor survival and it is used as an independent prognostic marker in primary gastric 
cancer (11). However, certain studies have shown that KLF4 expression is increased in breast ductal cell carcinoma (15) and oral squamous cell carcinoma (16) for as yet unknown reasons. Additionally, KLF4-knockout mouse studies have demonstrated that KLF4 may activate mucosal cell differentiation and induce precancerous changes in the stomach (17). These studies demonstrate the importance of KLF4 in various types of cancer, including gastric cancer, although the role of KLF4 in the staging and lymph node metastasis of gastric cancer remains to be determined.

$\beta$-catenin is a multifunctional protein involved in cadherin-mediated cell adhesion at the plasma membrane and transcriptional regulation in the nucleus (18), that is involved in a number of cell processes, including embryogenesis, tumorigenesis and tumor progression (19). In the cytoplasm of normal epithelium, the levels of $\beta$-catenin are regulated by the phosphorylation of its $\mathrm{N}$-terminal serine and threonine residues by the APC-Axin-GSK-3 $\beta$ complex. When $\beta$-catenin overexpression and its accumulation results in $\beta$-catenin-lymphoid enhancer factor (LEF)/T-cell factor (TCF) complex formation in the nucleus, $\beta$-catenin acts as a transcription factor, activating target genes such as cyclin-D1 and c-Myc (20). Thus, $\beta$-catenin has been confirmed as an oncogene in a variety of tumors $(21,22)$. In a number of types of aggressive and lethal cancer, the aberrant quantity and localization of $\beta$-catenin may weaken cell-cell junctions and promote the dedifferentiation, hyperproliferation, invasion and metastasis of tumor cells in different types of cancer, including gastric cancer, lung cancer and breast cancer (20). A high level of $\beta$-catenin activity is significantly correlated with the invasion and progression of a number of types of cancer $(23,24)$ and is used as an independent prognostic indicator for these cancers (25). KLF4 is known to be a novel antagonist of $\beta$-catenin in the nucleus. Crosstalk between KLF4 and $\beta$-catenin occurs in normal intestinal mucosae and colorectal cancer and is involved in the homeostasis of intestinal mucosae $(26,27)$. Therefore, the aim of this study was to investigate the expression levels of the KLF4 and $\beta$-catenin proteins in moderately differentiated gastric cancer tissues and determine whether their expression is associated with gastric cancer staging and lymph node metastasis.

\section{Materials and methods}

Tissue specimens. Forty-nine moderately differentiated specimens were obtained from gastric cancer patients who had undergone standard D2 radical gastric resection in the Department of Gastrointestinal Surgery, The First Affiliated Hospital of Chongqing Medical University (Chongqing, China) between November 2009 and May 2010. The clinicopathological data are shown in Table I. Staging of the tumors was performed according to the gastric cancer staging standard of the International Union Against Cancer (UICC). No patients underwent chemotherapy or radiotherapy prior to surgery. A matched distant non-cancerous sample $(5 \mathrm{~cm}$ away from the lesion) was also obtained from each patient and used as a control. Informed consent and permission to use their tissue in research was obtained from each patient. This study was performed in compliance with the Declaration of Helsinki with the approval of the Ethics Committee of Chongqing Medical University.
Immunohistochemistry. Formalin-fixed and paraffinembedded tumor specimens were prepared, cut into $5-\mu \mathrm{m}$ sections and mounted onto poly-L-lysine coated glass slides. The tissue sections were stained immunohistochemically with antigen retrieval methods (28) using a rabbit polyclonal antibody against human KLF4 (1:200 dilution, Abcam Biotechnology, Cambridge, UK) and a mouse monoclonal antibody against human $\beta$-catenin (1:200 dilution, Millipore Biotechnology, Boston, MA, USA). Positive staining was a reddish-brown precipitate in the nuclei and cytoplasm (29). The expression of KLF4 and $\beta$-catenin proteins was reviewed and scored according to the following grading system: staining intensity was categorized as negative (-), weak (+), moderate $(++)$ or strong $(+++)$. The percentage of staining was categorized as no staining $(-),<10 \%$ of tumor cell stained $(+)$, $10-40 \%(++), 40-70 \%(+++)$ and $>70 \%(++++)$. To simultaneously gauge the staining intensity and uniformity, the average values for the intensity in each slice were multiplied by the average values for the percentage area stained in each slice to derive a composite histoscore (histoscore $=$ intensity $\mathrm{x}$ area).

Protein extraction and western blotting. To confirm the quality of the antibody, we randomly selected seven cases from the 49 patients for protein extraction and western blot analysis. Samples $(50 \mathrm{mg}$ ) of each tumor and normal mucosa tissue were minced, washed three times with ice-cold PBS, gently centrifuged and soaked in $500 \mathrm{ml}$ of hypotonic buffer $(1 \mathrm{mM}$ $\mathrm{NaHCO}_{2}$ ) containing $2 \mathrm{mM}$ phenylmethanesulfonyl fluoride (PMSF) for $30 \mathrm{~min}$, followed by centrifugation at $15,000 \mathrm{x} \mathrm{g}$ for $15 \mathrm{~min}$. The supernatant was measured for protein concentration with a protein assay kit (Bio-Rad, Hercules, CA, USA). The protein samples were mixed with sample loading buffer (30\% glycerol, 6\% sodium-dodecylsulfate (SDS), $62.5 \mathrm{mM}$ Tris-HCl, pH 6.8), separated by $10 \%$ SDS-PAGE and transferred to a PVDF membrane (Millipore). The membrane was then blocked using 5\% skimmed milk, incubated with the appropriate primary antibodies and then incubated with secondary antibodies coupled with horseradish peroxidase (HRP) (Amersham, Arlington Heights, IL, USA). The protein bands were finally visualized with an enhanced chemiluminescence (ECL) reagent (Beyotime Institute of Biotechnology, Jiangsu, China).

Statistical analysis. Statistical analysis was performed using SPSS 17.0 for Windows (SPSS, Chicago, IL, USA) to assess the immunohistochemical data of KLF4 and $\beta$-catenin expression between gastric carcinoma and paired normal tissues with the Chi-square test and with the Student's t-test for western bloting data (data reported as the mean $\pm \mathrm{SD}$ ). The association between KLF4 and $\beta$-catenin was evaluated using the Spearman's correlation test. $\mathrm{P}<0.05$ was considered to indicate a statistically significant difference.

\section{Results}

Expression of KLF4 and $\beta$-catenin proteins in gastric cancer and distant normal tissue. In this study, we first assessed the expression of the KLF4 and $\beta$-catenin proteins in gastric cancer tissues and distant normal mucosae using western blotting. We found that the antibodies against the two 
A
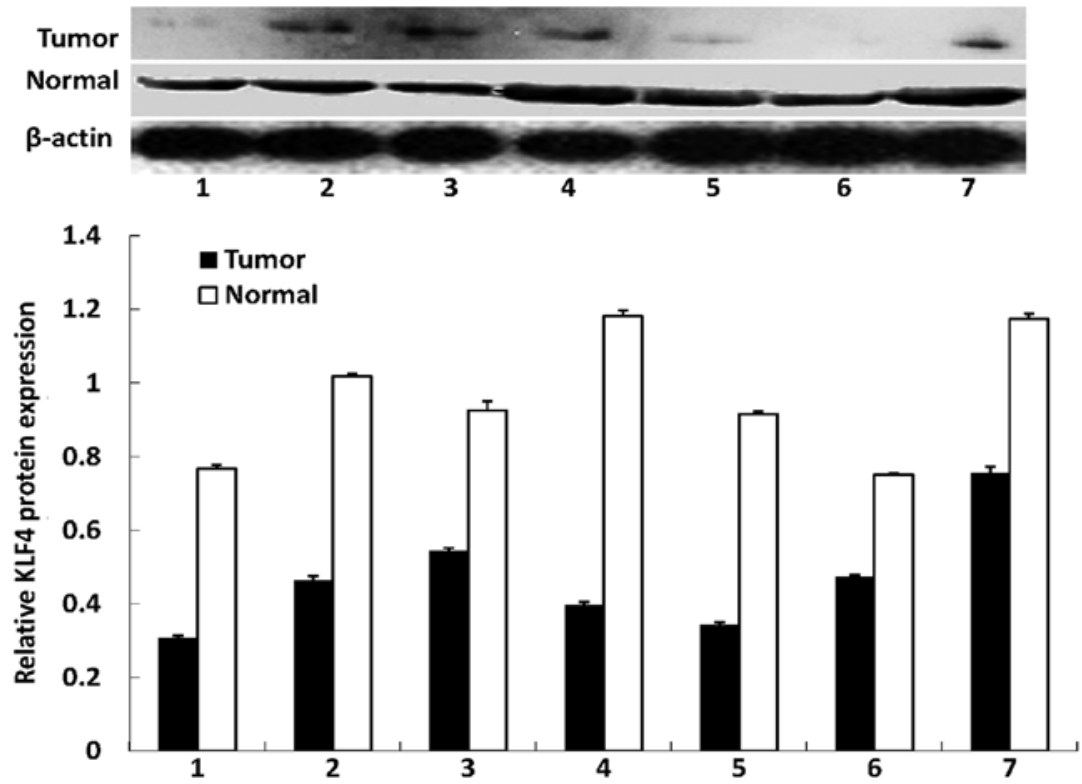

B
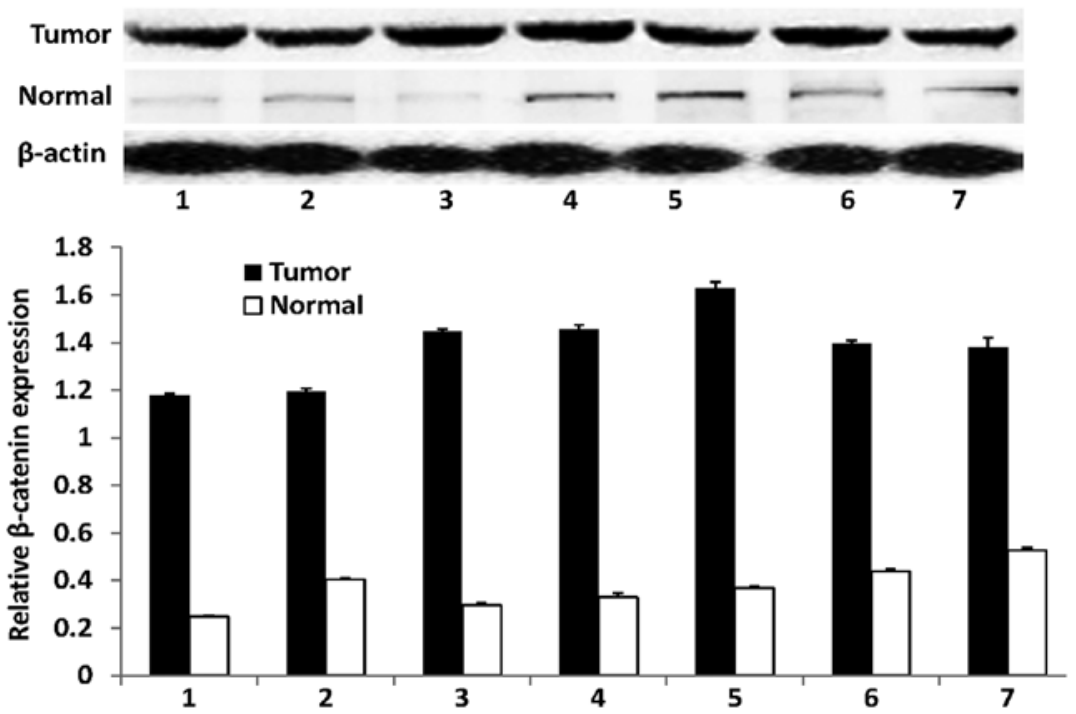

Figure 1. Western blotting of KLF4 and $\beta$-catenin in tumor tissues and matched distant non-cancerous tissues. (A) KLF4 expression. (B) $\beta$-catenin expression The bands were densitometrically scanned and $\beta$-actin was used as an internal control. The statistical analysis with the relative protein expression levels of KLF4 and $\beta$-catenin are shown in the graphs.

proteins were specific, indicating that they were suitable for immunohistochemistry. Western blotting revealed that KLF4 was highly expressed in the normal tissues, whereas all the tumor tissues had a reduced KLF4 expression. By contrast, the expression of the $\beta$-catenin protein was significantly increased in all tumor tissues compared with the distant normal mucosae (Fig. 1). The alteration of the expression of the two proteins was statistically significant $(\mathrm{P}<0.01$ for the two proteins).

Immunohistochemical analysis of KLF4 and $\beta$-catenin expression in gastric cancer tissues and distant normal mucosae. We analyzed the expression of the KLF4 and $\beta$-catenin proteins in paraffin-embedded cancer and paired-non-cancerous tissues from 49 cases of moderately differentiated gastric adenocarcinomas. The immunohistochemical data showed that $34 / 49(69.4 \%)$ gastric cancer tissues were positive for $\beta$-catenin, whereas only $22 / 49$ (44.90\%) of the distant normal mucosae expressed the $\beta$-catenin protein. Moreover, $\beta$-catenin was located in the nucleus. By contrast, positive staining of the KLF4 protein was found in only seven gastric cancer samples $(7 / 49,14.29 \%)$, whereas 40/49 (82.6\%) of the normal gastric mucosae expressed the KLF4 protein. Compared with the normal mucosae, KLF4 expression was significantly decreased in gastric cancer patients $(\mathrm{P}=0.0001$; Fig. 2). The increased $\beta$-catenin expression in gastric cancer tissues was also statistically significant ( $\mathrm{P}=0.014$; Fig. 3$)$. We then associated the expression of the two proteins with the clinicopathological data of the patients (Table I) and found that the decreased expression of KLF4, but increased levels of $\beta$-catenin, were associated with advanced tumor stage $(\mathrm{P}=0.041$ and $\mathrm{P}=0.034$, respectively). However, KLF4 and $\beta$-catenin expression in cancer tissues was not significantly associated with age $(\mathrm{P}=0.85$ and $\mathrm{P}=0.98$, respectively) or gender $(\mathrm{P}=0.686$ and $\mathrm{P}=0.78$, respectively). In addition, we 
Table I. Clinical characteristics and the expression of KLF4 and $\beta$-catenin in 49 gastric carcinoma patients.

\begin{tabular}{|c|c|c|c|c|c|c|c|}
\hline \multirow[b]{2}{*}{ Characteristic } & \multirow{2}{*}{$\begin{array}{l}\text { Number } \\
\text { of cases }\end{array}$} & \multicolumn{2}{|c|}{$\beta$-catenin } & \multirow[b]{2}{*}{ P-value } & \multicolumn{2}{|c|}{ KLF4 } & \multirow[b]{2}{*}{ P-value } \\
\hline & & Positive & Positive rate (\%) & & Positive & Positive rate (\%) & \\
\hline \multicolumn{8}{|l|}{ Age (years) } \\
\hline$\leq 60$ & 23 & 16 & 69.57 & & 3 & 13.04 & \\
\hline$>60$ & 26 & 18 & 69.23 & 0.98 & 4 & 15.38 & 0.85 \\
\hline \multicolumn{8}{|l|}{ Gender } \\
\hline Female & 15 & 10 & 66.67 & & 2 & 13.33 & \\
\hline Male & 34 & 24 & 70.59 & 0.78 & 5 & 14.71 & 0.686 \\
\hline \multicolumn{8}{|l|}{ Stage } \\
\hline $\mathrm{I}+\mathrm{II}$ & 17 & 10 & 58.82 & & 5 & 29.40 & \\
\hline III & 32 & 24 & 75.00 & 0.242 & 2 & 6.25 & 0.041 \\
\hline \multicolumn{8}{|c|}{ Lymph node metastasis } \\
\hline Present & 36 & 28 & 82.35 & & 7 & 16.67 & \\
\hline Absent & 13 & 6 & 40.15 & 0.034 & 0 & 7.69 & 0.086 \\
\hline
\end{tabular}

Staging was performed according to the gastric cancer staging standard of the International Union Against Cancer (UICC).

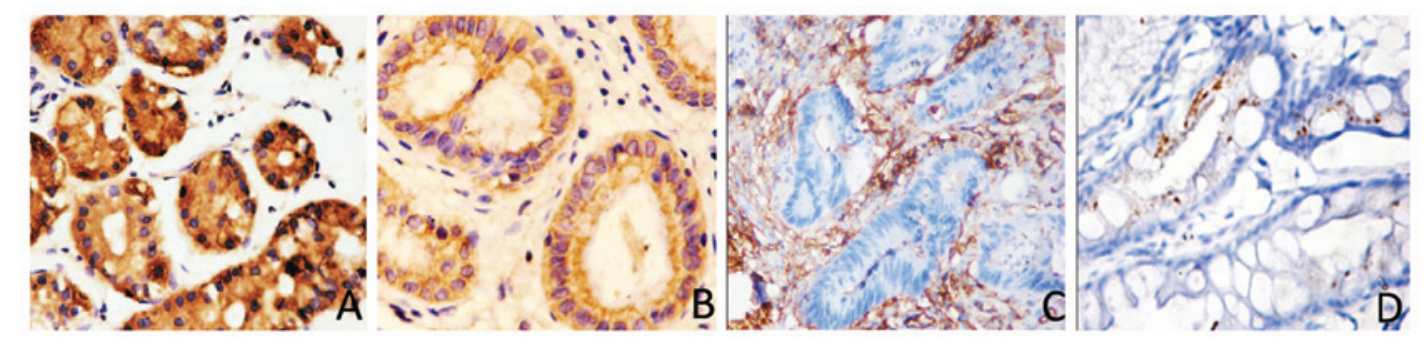

Figure 2. Expression of KLF4 protein in human gastric adenocarcinoma and matched distant non-cancerous tissues by immunohistochemistry. (A) Strong KLF4 expression (+++) in distant non-cancerous tissues. (B) Positive KLF4 expression (++) in different stages of gastric cancer. (C) Weakly positive KLF4 expression (+) in different stages of gastric cancer. (D) Negative KLF4 expression (-) in different stages of gastric cancer. Original magnification, x200.

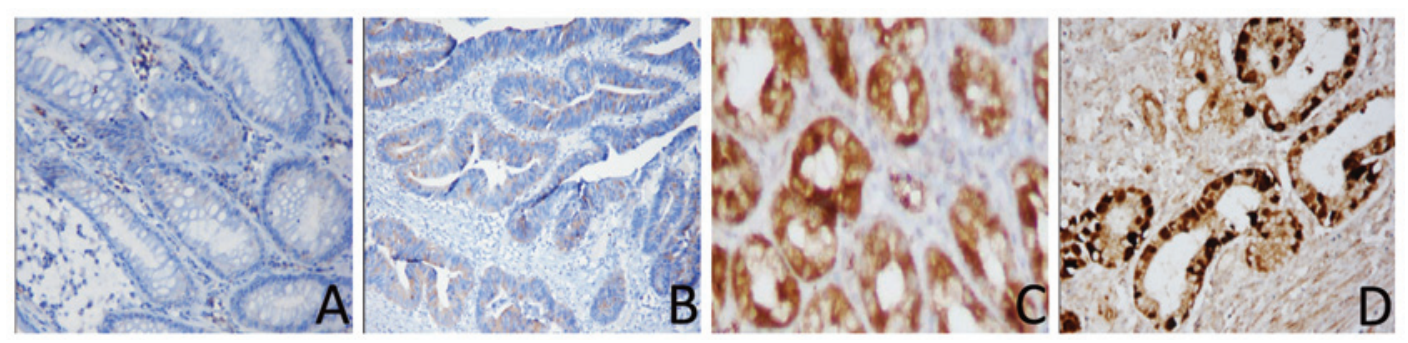

Figure 3. Expression of $\beta$-catenin protein in human gastric adenocarcinoma and matched distant non-cancerous tissues by immunohistochemistry. (A) Weak $\beta$-catenin expression (+) in distant non-cancerous tissues. (B) Weak $\beta$-catenin expression (+) in different stages of gastric cancer. (C) Weakly positive $\beta$-catenin expression (++) in different stages of gastric cancer. (D) Strongly positive $\beta$-catenin expression (+++) in different stages of gastric cancer. Original magnification, $\mathrm{x} 200$

found a significant inverse correlation between KLF4 and $\beta$-catenin expression in moderately differentiated human gastric cancers $\left(\mathrm{r}_{\mathrm{s}}=-0.488 ; \mathrm{P}<0.001\right)$.

\section{Discussion}

In this study, we analyzed the expression of the KLF4 and $\beta$-catenin proteins in gastric cancer and corresponding normal tissues. The immunohistochemical data have shown that $\beta$-catenin expression was upregulated in gastric cancer tissues compared with distant normal mucosae, whereas the expression of KLF4 protein was significantly decreased in gastric cancer samples compared with normal gastric mucosae. The altered expression of KLF4 and $\beta$-catenin was associated with the advanced tumor stage of gastric cancer. In addition, the expression of the KLF4 protein was inversely correlated with 
that of $\beta$-catenin in moderately differentiated human gastric cancers. The data from the current study demonstrate that the expression of the $\beta$-catenin protein is significantly increased, whereas that of the KLF4 protein is markedly decreased, in gastric cancer tissues, and that the expression of KLF4 is inversely associated with that of $\beta$-catenin. The altered expression of the two proteins is associated with advanced tumor stage in gastric cancer. More studies are needed to verify the value of these two proteins as biomarkers for the prediction of gastric cancer progression.

The results of previous studies have shown that KLF4 is highly expressed in gastrointestinal epithelial cells and is associated with growth arrest (8), by blocking G1/S progression of the cell cycle, and terminal differentiation $(30,31)$. The studies published currently have speculated that KLF4 is involved in the maintenance of gastric mucosa homeostasis (11). However, depending upon molecular events, KLF4 may act as either a tumor suppressor gene or an oncogene in different cells $(32,33)$. For example, the number of goblet cells in the colon is substantially decreased in KLF4-null mice (34), which may be a significant event in colorectal tumor development. Nevertheless, the levels of KLF4 RNA and protein are significantly reduced in the dysplastic epithelium, adenomatous polyps and colon cancers, indicating the involvement of KLF4 in colorectal carcinogenesis (35). On the other hand, an inverse correlation has also been found between KLF4 and the size of multiple intestinal adenomas in mice (36). The alteration of KLF4 expression leads to aberrant proliferation and differentiation in gastric and colonic epithelium (17,34). Low levels or the loss of KLF4 expression in a number of types of cancer (9-14) have been reported and increasing evidence suggests that KLF4 is a putative tumor suppressor in the digestive tract, including the stomach and colon $(9,11)$. KLF4 knockout mice exhibit defects in gastric differentiation and have precancerous changes in the stomach $(11,17)$. In addition, the overexpression of KLF4 in a colon cancer cell line induced colon cancer cell growth arrest (30), reduced colony formation, cell migration and invasion and repressed $\beta$-catenin transcription in colon cancer HT29 cells (37). These results suggest that KLF4 is a tumor suppressor gene and is involved in the progression and development of gastric tumors. In the current study, we found that the KLF4 protein was predominantly expressed in gastric non-neoplastic epithelium, but was substantially decreased or lost in gastric tumor specimens.

$\beta$-catenin is a crucial part of the cell-cell adhesion complex associated with cadherins (e.g., $\alpha-, \beta$ - and E-cadherin). $\beta$-catenin has been found to mediate intercellular adhesion (18), as well as to regulate cell growth and differentiation. $\beta$-catenin is a key downstream molecule in the Wnt signaling pathway and binds to the TCF/LEF transcription factors to regulate and activate the transcription of target genes that are involved in embryo development, tissue self-renewal and cancer (38). $\beta$-catenin is pivotal in intracellular signaling and is a key element in one of the most significant pathways in epithelial carcinogenesis $(39,40)$. $\beta$-catenin has been identified as an oncogene in a variety of tumors in numerous previous studies $(23,41)$. In a number of types of aggressive and lethal cancer, aberrant $\beta$-catenin expression may weaken cell-cell junctions and promote carcinoma cell dedifferentiation, hyperproliferation, invasion and metastasis, characteristics that are commonly found in a number of tumors, including gastric, lung and breast cancers (42). A high level of $\beta$-catenin activity is significantly correlated with the invasiveness and progression of numerous tumors, including gastric cancer $(8,23,24)$. Previous studies have confirmed that $\beta$-catenin is an independent prognostic indicator for these carcinomas and is closely correlated with tumor progression $(25,41)$. However, it has been reported that KLF4 regulates Wnt/ $\beta$-catenin signaling, which is significant in the homeostasis of the normal intestine, and indicates important implications in cancer research $(26,27)$. In the present study, we investigated the expression levels of KLF4 and $\beta$-catenin and the potential association between KLF4 and $\beta$-catenin expression in human gastric cancer and corresponding normal tissue. This study identified patterns in the expression of KLF4 and $\beta$-catenin. Notably, the expression of the $\beta$-catenin protein was found to be inversely associated with KLF4 expression, suggesting that a decrease in the expression of KLF4 reduces its ability to inhibit $\beta$-catenin expression levels. The overexpression of $\beta$-catenin is commonly observed in colorectal cancers, while KLF4 expression is decreased in a variety of types of cancer (9-14). Our data are consistent with those published in other studies (9-14).

In the current study, the altered expression of these two proteins was associated with certain clinicopathological parameters. For example, reduced KLF4 expression was associated with advanced TNM stage of gastric cancer, while the expression of $\beta$-catenin was associated with lymph node metastasis of gastric cancer. To the best of our knowledge, this is the first study showing an inverse correlation between KLF4 and $\beta$-catenin expression in gastric cancer, which may lead to a fuller understanding of the interaction between KLF4 and $\beta$-catenin, thereby widening diagnosis and treatment options for gastric cancer. Although novel, these data should be further verified with a larger sample size. In addition, future studies are required to investigate the molecular link between KLF4 and $\beta$-catenin proteins and further evaluate them as potential biomarkers for gastric cancer development and progression.

\section{Acknowledgements}

This study was supported in part by a grant from the 2010 Annual Medical Research Projects of the Chongqing Municipal Health Bureau (no. 2010-1-19).

\section{References}

1. Parkin DM, Bray F, Ferlay J and Pisani P: Global cancer statistics, 2002. CA Cancer J Clin 55: 74-108, 2005.

2. Murray D, Horgan G, Macmathuna P and Doran P: NET1mediated RhoA activation facilitates lysophosphatidic acid-induced cell migration and invasion in gastric cancer. $\mathrm{Br} \mathrm{J}$ Cancer 99: 1322-1329, 2008.

3. Tahara E: Molecular aspects of invasion and metastasis of stomach cancer. Verh Dtsch Ges Pathol 84: 43-49, 2000.

4. Sud R, Wells D, Talbot IC and Delhanty JD: Genetic alterations in gastric cancers from British patients. Cancer Genet Cytogenet 126: 111-119, 2001

5. Anderson KP, Kern CB, Crable SC and Lingrel JB: Isolation of a gene encoding a functional zinc finger protein homologous to erythroid Krüppel-like factor: identification of a new multigene family. Mol Cell Biol 15: 5957-5965, 1995.

6. Kanai M, Wei D, Li Q, et al: Loss of Krüppel-like factor 4 expression contributes to $\mathrm{Sp} 1$ overexpression and human gastric cancer development and progression. Clin Cancer Res 12: 6395-6402, 2006. 
7. McConnell BB, Ghaleb AM, Nandan MO and Yang VW: The diverse functions of Krüppel-like factors 4 and 5 in epithelial biology and pathobiology. Bioessays 29: 549-557, 2007.

8. Shields JM, Christy RJ and Yang VW: Identification and characterization of a gene encoding a gut-enriched Krüppel-like factor expressed during growth arrest. J Biol Chem 271: 20009-20017, 1996.

9. Zhao W, Hisamuddin IM, Nandan MO, Babbin BA, Lamb NE and Yang VW: Identification of Krüppel-like factor 4 as a potential tumor suppressor gene in colorectal cancer. Oncogene 23: 395-402, 2004.

10. Ton-That H, Kaestner KH, Shields JM, Mahatanankoon CS and Yang VW: Expression of the gut-enriched Krüppel-like factor gene during development and intestinal tumorigenesis. FEBS Lett 419: 239-243, 1997.

11. Wei D, Gong W, Kanai M, et al: Drastic down-regulation of Krüppel-like factor 4 expression is critical in human gastric cancer development and progression. Cancer Res 65: 2746-2754, 2005.

12. Yang Y, Goldstein BG, Chao HH and Katz JP: KLF4 and KLF5 regulate proliferation, apoptosis and invasion in esophageal cancer cells. Cancer Biol Ther 4: 1216-1221, 2005.

13. Schulz WA and Hatina J: Epigenetics of prostate cancer: beyond DNA methylation. J Cell Mol Med 10: 100-125, 2006.

14. $\mathrm{Hu} \mathrm{W}$, Hofstetter WL, Li H, et al: Putative tumor-suppressive function of Kruppel-like factor 4 in primary lung carcinoma. Clin Cancer Res 15: 5688-5695, 2009.

15. Foster KW, Frost AR, McKie-Bell P, et al: Increase of GKLF messenger RNA and protein expression during progression of breast cancer. Cancer Res 60: 6488-6495, 2000.

16. Foster KW, Ren S, Louro ID, et al: Oncogene expression cloning by retroviral transduction of adenovirus E1A-immortalized rat kidney RK3E cells: transformation of a host with epithelial features by c-MYC and the zinc finger protein GKLF. Cell Growth Differ 10: 423-434, 1999.

17. Katz JP, Perreault N, Goldstein BG, et al: Loss of Klf4 in mice causes altered proliferation and differentiation and precancerous changes in the adult stomach. Gastroenterology 128: 935-945, 2005 .

18. Ozawa M, Ringwald M and Kemler R: Uvomorulin-catenin complex formation is regulated by a specific domain in the cytoplasmic region of the cell adhesion molecule. Proc Natl Acad Sci USA 87: 4246-4250, 1990.

19. Hoppler S and Kavanagh CL: Wnt signalling: variety at the core. J Cell Sci 120: 385-393, 2007.

20. Conacci-Sorrell M, Simcha I, Ben-Yedidia T, Blechman J, Savagner P and Ben-Ze'ev A: Autoregulation of E-cadherin expression by cadherin-cadherin interactions: the roles of beta-catenin signaling, Slug, and MAPK. J Cell Biol 163: 847-857, 2003.

21. Bieker JJ: Krüppel-like factors: three fingers in many pies. J Biol Chem 276: 34355-34358, 2001.

22. Shie JL, Chen ZY, O'Brien MJ, Pestell RG, Lee ME and Tseng CC: Role of gut-enriched Krüppel-like factor in colonic cell growth and differentiation. Am J Physiol Gastrointest Liver Physiol 279: G806-G814, 2000.

23. Choi YS, Shim YM, Kim SH, et al: Prognostic significance of E-cadherin and beta-catenin in resected stage I non-small cell lung cancer. Eur J Cardiothorac Surg 24: 441-449, 2003.

24. Resnick MB, Routhier J, Konkin T, Sabo E and Pricolo VE: Epidermal growth factor receptor, c-MET, beta-catenin, and p53 expression as prognostic indicators in stage II colon cancer: a tissue microarray study. Clin Cancer Res 10: 3069-3075, 2004.
25. Wong SC, Lo ES, Lee KC, Chan JK and Hsiao WL: Prognostic and diagnostic significance of beta-catenin nuclear immunostaining in colorectal cancer. Clin Cancer Res 10: 1401-1408, 2004

26. Zhang W, Chen X, Kato Y, et al: Novel cross talk of Kruppel-like factor 4 and beta-catenin regulates normal intestinal homeostasis and tumor repression. Mol Cell Biol 26: 2055-2064, 2006.

27. Evans PM, Chen X, Zhang W and Liu C: KLF4 interacts with beta-catenin/TCF4 and blocks p300/CBP recruitment by beta-catenin. Mol Cell Biol 30: 372-381, 2010.

28. Ciaparrone M, Yamamoto H, Yao Y, et al: Localization and expression of p27KIP1 in multistage colorectal carcinogenesis. Cancer Res 58: 114-122, 1998.

29. Wang L, Wei D, Huang S, et al: Transcription factor Spl expression is a significant predictor of survival in human gastric cancer. Clin Cancer Res 9: 6371-6380, 2003.

30. Chen X, Johns DC, Geiman DE, et al: Krüppel-like factor 4 (gut-enriched Krüppel-like factor) inhibits cell proliferation by blocking G1/S progression of the cell cycle. J Biol Chem 276: 30423-30428, 2001.

31. Yoon HS and Yang VW: Requirement of Krüppel-like factor 4 in preventing entry into mitosis following DNA damage. J Biol Chem 279: 5035-5041, 2004.

32. Rowland BD and Peeper DS: KLF4, p21 and context-dependent opposing forces in cancer. Nat Rev Cancer 6: 11-23, 2006.

33. Rowland BD, Bernards R and Peeper DS: The KLF4 tumour suppressor is a transcriptional repressor of p53 that acts as a context-dependent oncogene. Nat Cell Biol 7: 1074-1082, 2005.

34. Katz JP, Perreault N, Goldstein BG, et al: The zinc-finger transcription factor Klf4 is required for terminal differentiation of goblet cells in the colon. Development 129: 2619-2628, 2002.

35. Shie JL, Chen ZY, Fu M, Pestell RG and Tseng CC: Gut-enriched Krüppel-like factor represses cyclin D1 promoter activity through Sp1 motif. Nucleic Acids Res 28: 2969-2976, 2000.

36. Dang DT, Bachman KE, Mahatan CS, Dang LH, Giardiello FM and Yang VW: Decreased expression of the gut-enriched Krüppellike factor gene in intestinal adenomas of multiple intestinal neoplasia mice and in colonic adenomas of familial adenomatous polyposis patients. FEBS Lett 476: 203-207, 2000.

37. Stone CD, Chen ZY and Tseng CC: Gut-enriched Krüppel-like factor regulates colonic cell growth through APC/beta-catenin pathway. FEBS Lett 530: 147-152, 2002.

38. Logan CY and Nusse R: The Wnt signaling pathway in development and disease. Annu Rev Cell Dev Biol 20: 781-810, 2004.

39. Hervieu V, Lepinasse F, Gouysse G, et al: Expression of betacatenin in gastroenteropancreatic endocrine tumours: a study of 229 cases. J Clin Pathol 59: 1300-1304, 2006.

40. Kanwar SS, Yu Y, Nautiyal J, Patel BB and Majumdar AP: The Wnt/beta-catenin pathway regulates growth and maintenance of colonospheres. Mol Cancer 9: 212, 2010

41. Lin SY, Xia W, Wang JC, et al: Beta-catenin, a novel prognostic marker for breast cancer: its roles in cyclin D1 expression and cancer progression. Proc Natl Acad Sci USA 97: 4262-4266, 2000.

42. Bianchi F, Hu J, Pelosi G, et al: Lung cancers detected by screening with spiral computed tomography have a malignant phenotype when analyzed by cDNA microarray. Clin Cancer Res 10: 6023-6028, 2004 